\title{
Correlation between the elevated uric acid levels and circulating renin-angiotensin-aldosterone system activation in patients with atrial fibrillation
}

\author{
Xue-Dong Wang ${ }^{1}$, Jing Liu ${ }^{1}$, Yu-Chen Zhang ${ }^{2}$, Yu Wang', Yan Wang ${ }^{1}$, Dan Ma ${ }^{1}$ \\ ${ }^{1}$ Coronary Care Unit, Beijing Hepingli Hospital, Beijing, China; ${ }^{2}$ Department of Cardiology, Beijing Anzhen Hospital Affiliated to Capital Medical \\ University, Beijing, China \\ Contributions: (I) Conception and design: XD Wang, J Liu; (II) Administrative support: XD Wang; (III) Provision of study materials or patients: YC \\ Zhang, D Ma; (IV) Collection and assembly of data: XD Wang, J Liu; (V) Data analysis and interpretation: Yu Wang, Yan Wang; (VI) Manuscript \\ writing: All authors; (VII) Final approval of manuscript: All authors. \\ Correspondence to: Xue-Dong Wang, MD. Coronary Care Unit, Beijing Hepingli Hospital, No. 18 of Hepingli North Street, Dongcheng District, \\ Beijing 100013, China. Email: wangxd_fjit@163.com.
}

Background: The aim of the present study was to investigate the correlation between the elevated uric acid (UA) levels and activation of the circulating renin-angiotensin-aldosterone system (RAAS) in patients with atrial fibrillation (AF).

Methods: A total of 233 outpatients and inpatients of the Cardiology Department from January 1, 2019, to December 31, 2019, were selected and divided into the sinus rhythm group (SR) with 84 cases, the paroxysmal AF group (pAF) with 76 cases, and the persistent AF group (PAF) with 73 patients. The general clinical data and the serum levels of UA of the enrolled patients were collected, and the radioimmunoassay was adopted to detect the levels of renin (Renin), angiotensin II (Ang II), and aldosterone (Ald).

Results: Renin, AngII, Ald, and UA in the PAF group were significantly higher than those in the pAF group, and the levels of the above indicators in the pAF group were significantly higher than those in the SR group $(\mathrm{P}<0.001)$. The left atrium anteroposterior diameter $(\mathrm{LAD})$ and the left ventricular end-diastolic diameter (LVEDD) were significantly increased in the PAF group $(\mathrm{P}<0.001)$. The Pearson correlation analysis showed that the levels of the high sensitivity C-reactive protein (hsCRP), AngII Renin, Ald, LVEDD, and LAD were positively correlated with the serum levels of UA ( $r=0.174,0.273,0.34,0.385,0.138$, respectively, $\mathrm{P}<0.05$ in all). The left ventricular ejection fraction (LVEF) was negatively correlated with the UA level $(r=-0.177, \mathrm{P}<0.05)$. Multiple linear regression analysis showed that $\mathrm{UA}(\beta=0.103)$ and LAD $(\beta=2.162)$ were independent risk factors for Renin. The independent risk factor for Ang II was UA $(\beta=0.167)$. The independent risk factor for Ald was UA $(\beta=0.283)$ and $\operatorname{LAD}(\beta=8.721)(\mathrm{P}<0.05)$.

Conclusions: Elevated UA might cause excessive activation of the RAAS, aggravate the oxidative stress, and participate in the atrial remodeling, thereby promoting the occurrence and persistence of AF.

Keywords: Uric acid (UA); atrial fibrillation (AF); renin-angiotensin-aldosterone system (RAAS)

Submitted Sep 28, 2020. Accepted for publication Dec 14, 2020.

doi: $10.21037 / \mathrm{cdt}-20-830$

View this article at: http://dx.doi.org/10.21037/cdt-20-830

\section{Introduction}

Atrial fibrillation (AF) is the most common non-benign arrhythmia in clinical practice, and the prevalence, fatality (heart failure), and disability (stroke) rates remain high (1-3). The atrial remodeling is the core part of AF, and the activation of the renin-angiotensin-aldosterone system (RAAS) is the key to the atrial remodeling (4). As a multi-factorial disease, serum uric acid (UA) is also one 
of the independent related factors of AF (5). Studies $(6,7)$ have confirmed that the increased serum levels of UA are correlated with the activation of the circulating RAAS in patients with hypertension. Therefore, we speculated that UA might also be involved in the pathophysiological process of atrial remodeling by activating the circulating RAAS, thereby promoting the occurrence and development of AF. The aim of the present study was to verify the above speculation. We present the following article in accordance with the MDAR checklist and the STROBE reporting checklist (available at: http://dx.doi.org/10.21037/cdt-20830).

\section{Methods}

The study was conducted in accordance with the Declaration of Helsinki (as revised in 2013). The study was approved by Ethics Committee of Beijing Hepingli Hospital (No. 20190109) and informed consent was taken from all the patients.

\section{Study objects}

The study objects were selected from the outpatients and those who came for health examination to Beijing Anzhen Hospital and Beijing Hepingli Hospital from January 1, 2019, to December 31, 2019. A total of 233 subjects were enrolled, with 116 males and 117 females. The ages ranged between $42-89$ years with an average age of $64 \pm 8.0$ years. The subjects were divided into three groups, of which 84 cases were in the sinus rhythm group (SR), 76 cases were in the paroxysmal AF group ( $\mathrm{pAF}$, with the duration $\leq 7$ days and could be terminated spontaneously), and the persistent AF group (PAF, with the duration $>7$ days and non-selflimiting) with 73 cases (8). The exclusion criteria were those with gout, tumor, infection, heart failure, chronic kidney disease, diabetes, blood system disease, and use of thiazide diuretics.

\section{Study methods}

\section{General data}

The data including the age, gender, systolic blood pressure (SBP), diastolic blood pressure (DBP), body mass index $\left[\mathrm{BMI}=\right.$ weight $(\mathrm{kg}) /$ height $\left.^{2}\left(\mathrm{~m}^{2}\right)\right]$, the previous history of hypertension, administration of $\beta$-blockers, and administration of angiotensin-converting enzyme inhibitor (ACEI)/angiotensin receptor antagonist (ARB) were collected.

\section{The laboratory and auxiliary examinations}

After the patients were enrolled, those taking ACEI/ ARBs should stop these for at least two weeks. Five $\mathrm{mL}$ of venous blood from the anterior elbow was drawn in the early morning while sitting and under a fasting condition. The automatic biochemical analyzer (LX20 biochemical analyzer, Beckman Coulter, USA) was used to determine the serum UA, aspartate aminotransferase (AST), blood creatinine (CREA), and other biochemical indicators. Radioimmunoassay was used to determine the plasma levels of renin (Renin), Angiotensin II (Ang II), and Aldosterone (Ald). Echocardiography was adopted to record the left atrium anteroposterior diameter (LAD), left ventricular end-diastolic diameter (LVEDD), and left ventricular ejection fraction (LVEF).

\section{Statistics analysis}

The measurement data were expressed by $\left(\bar{x}_{ \pm s}\right)$. The $t$-test was used to compare the means of two samples, and the $\chi^{2}$ test was used to compare the composition ratio between the two samples. One-way analysis of variance (oneway ANOVA) was used for the comparison of the means among multiple samples, the SNK (Student-NewmanKeuls) method was used for comparison between groups, and the Kruskal-Wallis test was used for the comparison of composition ratios among multiple samples. Pearson correlation analysis was adopted to investigate the correlation between the UA level and various clinical laboratory indicators. A multiple linear regression model was used for multivariate analysis. The SPSS for Windows 21.0 statistical software was used for data analysis, and $\mathrm{P}<0.05$ was considered statistically significant.

\section{Results}

\section{Comparison of the general characteristics and results of laboratory tests among the three groups}

As demonstrated in Table 1, in terms of the general characteristics, the age in the PAF group was significantly higher than that in the $\mathrm{SR}$ and the pAF group $(\mathrm{P}<0.001)$. For other indicators such as the SBP, DBP, BMI, and the proportion of drugs administration, there was no statistical difference among the three groups in the analysis of variance $(\mathrm{P}>0.05$ in all $)$. 
Table 1 Comparison of the general characteristics and results of laboratory test among the three groups

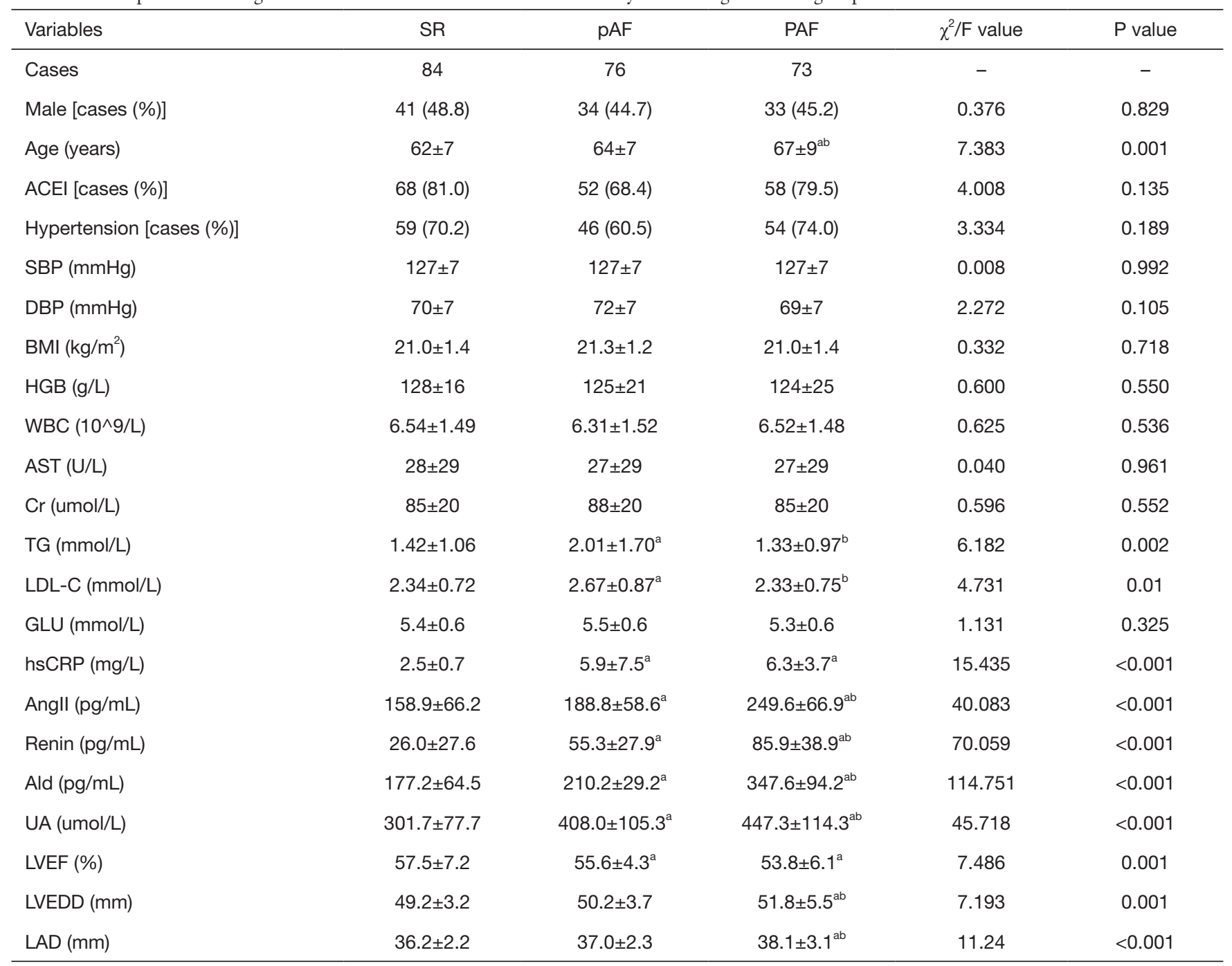

Comparison with SR group: ${ }^{a}, \mathrm{P}<0.05$; compared with pAF group: ${ }^{\mathrm{b}}, \mathrm{P}<0.05 .1 \mathrm{mmHg}=0.133 \mathrm{kPa}$; ACEl, angiotensin converting enzyme inhibitor; SBP, systolic blood pressure; DBP, diastolic blood pressure; BMI, body mass index; HGB, hemoglobin; WBC, white blood cell; AST, aspartate aminotransferase; Cr, Creatinine; TG, triglyceride; LDL-C, low-density lipoprotein cholesterol; GLU, fasting blood glucose; hsCRP, high sensitivity C-reactive protein; Angll, angiotensin II; Ald, aldosterone; UA, uric acid; LVEF, left ventricular ejection fraction; LVEDD, left ventricular end-diastolic diameter; LAD, left atrium diameter.

In terms of the results of laboratory tests, Renin, Ang II, Ald, and UA in the PAF group were significantly higher than those in the pAF group $(\mathrm{P}<0.001)$, and the above indicators in the pAF group was significantly higher than those in the SR group $(\mathrm{P}<0.001)$. LVEDD and LAD were not significantly different between the SR group and the pAF group, but these indicators were significantly higher in the PAF group $(\mathrm{P}<0.001)$. There was no significant difference in the hsCRP and LVEF between the PAF group and the pAF group, but the hsCRP level in both the PAF group and pAF group was higher than that in the SR group, while the LVEF in both the PAF group and pAF group was lower than that in the SR group $(\mathrm{P}<0.001)$. The levels of triglyceride (TG) and low-density lipoprotein cholesterol (LDL-C) were significantly higher in the pAF group than those in the SR group but were significantly lower in the PAF group than in the pAF group $(\mathrm{P}<0.05)$. Other indicators, such as the hemoglobin level and CREA, were not statistically different among the three groups $(\mathrm{P}>0.05$ in all). 
Table 2 Pearson correlation analysis

\begin{tabular}{lccccccccc}
\hline Variable & TG & LDL & hsCRP & Angll & Renin & Ald & LVEF & LVEDD & LAD \\
\hline R value & 0.091 & -0.095 & 0.174 & 0.273 & 0.340 & 0.385 & -0.177 & 0.189 & 0.138 \\
P value & 0.164 & 0.146 & 0.008 & $<0.001$ & $<0.001$ & $<0.001$ & 0.007 & 0.004 & 0.035 \\
\hline
\end{tabular}

TG, triglyceride; LDL, low-density lipoprotein cholesterol; hsCRP, high sensitivity C-reactive protein; Angll, angiotensin II; Ald, aldosterone; LVEF, left ventricular ejection fraction; LVEDD, left ventricular end-diastolic diameter; LAD, left atrium diameter.

Table 3 Multiple linear regression analysis of the influencing factors of Renin, AngII, Ald dependent variable/independent variable

\begin{tabular}{lccccc}
\hline Dependent variable/independent variable & B & SE & B' & T value & P value \\
\hline Renin/UA & 0.103 & 0.022 & 0.301 & 4.667 & 2.237 \\
Renin/LAD & 2.162 & 0.966 & 0.143 & 0.026 \\
Angll/UA & 0.167 & 0.042 & 0.265 & 4.002 & 5.202 \\
Ald/UA & 0.283 & 0.054 & 0.320 & $<.001$ \\
Ald/LAD & 8.721 & 2.389 & 0.223 & 3.650 & $<0.001$ \\
\hline
\end{tabular}

B: the non-standardized regression coefficient, B': the standardized regression coefficient. UA, uric acid; LAD, left atrium diameter; Angll, angiotensin II; Ald, aldosterone.

\section{Analysis of the $U A$ related factors}

Age, TG, LDL, hsCRP, AngII, Renin, Ald, LVEF, LVEDD, and LAD were selected as the independent variables, and Pearson correlation analysis with the serum levels of UA was performed. The results showed that hsCRP, AngII, Renin, Ald, LVEDD, and LAD were positively correlated with the serum levels of UA $(r=0.174,0.273,0.34,0.385$, $0.138, \mathrm{P}<0.05$ in all), and LVEF was negatively correlated with UA levels $(\mathrm{r}=-0.177, \mathrm{P}<0.05$, Table 2$)$.

\section{Multiple linear regression analysis of the related factors of RAAS activation}

With Renin, Ang II, and Ald as the dependent variables, and age, TG, LDL, hsCRP, UA, LVEF, LVEDD, and LAD as the independent variables, multiple linear regression analysis was performed. The results revealed that UA $(\beta=0.103)$ and $\operatorname{LAD}(\beta=2.162)$ were independent risk factors for Renin. The independent risk factor for Ang II was UA $(\beta=0.167)$. The independent risk factors for Ald were UA $(\beta=0.283)$ and $\operatorname{LAD}(B=8.721)(\mathrm{P}<0.05$ in all $)$, as shown in Table 3.

\section{Discussion}

As a multi-etiological, multi-factorial disease, the relationship between $\mathrm{AF}$ and the serum levels of $\mathrm{UA}$ has been one of the hot research fields in recent years $(9,10)$. A previous study (11) has shown that UA might be one of the independent risk factors for $\mathrm{AF}$, but the mechanism of causing AF is unclear and tends to be correlated with the inflammation activation and increased oxidative stress caused by elevated serum levels of UA (12). In patients with $\mathrm{AF}$, the relationship between the level of $\mathrm{UA}$ and the activation of the RAAS system has not been reported in domestic and foreign literature.

In the present study, 233 patients were enrolled and divided into three groups of SR, pAF, and PAF according to the existence and time course of AF. Univariate analysis found that the levels of Renin, AngII, Ald, and UA in the PAF group were significantly higher than those in the SR group and the pAF group, while the levels of the above indicators in the pAF group were significantly higher than those in the SR group. This suggested, on the one hand, that the RAAS activation and UA might be involved in the occurrence of AF. On the other hand, it indicated that the continuous increase in the degree of RAAS activation and the continuous rise in the level of UA might participate in the development of AF.

The univariate analysis of the present study also found that LVEDD and LAD in the PAF group were significantly higher than those in the SR group and the pAF group, and hsCRP and LVEF also had significant changes in the PAF 
group compared with the SR group. These results indicated that in the PAF stage, due to the long-term loss of atrial contraction, the left atrial pressure load would increase with the left atrium enlargement and fibrosis, and finally, the structural remodeling of the left atrium and left ventricle would occur, which might further promote the selfmaintenance of AF. With the left ventricular remodeling, the systolic function declines. This process is accompanied by the activation of inflammation, which is manifested by a decrease in LVEF and an increase in hsCRP (13).

Correlation analysis in the present study found that during the process of AF from absence to presence, from paroxysmal to continuous, the serum levels of UA demonstrated varying degrees of correlation with hsCRP, AngII, Renin, Ald, LVEDD, LAD, and LVEF. This further suggested that the serum UA, RAAS activation, inflammation, and cardiac structural remodeling might be closely correlated with the occurrence and development of $\mathrm{AF}(14,15)$.

In multivariate analysis, the multiple linear regression model was adopted. It was found that UA was not only an independent risk factor for Renin but also an independent risk factor for Ang II and Ald, suggesting that the UA level might be closely correlated with the activation of RAAS, which meant that during the occurrence and development of AF, the role of UA was likely to be realized through the RAAS activation. With the RAAS activation, the increased production of Renin and Ang II can regulate the $\mathrm{Ca}^{2+}$ and $\mathrm{K}^{+}$currents in the cardiomyocytes, shorten the duration of action potentials, and trigger the atrial electrical remodeling. In addition, the RAAS activation can lead to atrial remodeling $(16,17)$. Animal experiments found that a small dose of Ang II infusion for 2-6 weeks could cause myocardial fibrosis. Ald has a more significant effect on $\mathrm{AF}$, which is the strongest known substance that can promote the myocardial fibrosis and is also correlated with myocardial inflammation and hypertrophy of myocardial cells. The incidence of AF in patients with primary aldosteronism is 12 times that of patients with ordinary hypertension, and the main mechanism is that Ald might promote atrial remodeling $(18,19)$.

The mechanisms for UA in RAAS activation were as follows: (I) up-regulation of the mRNA expression of angiotensinogen. UA enters the vascular smooth muscle cells through the uptake of organic anion transporters, increases the expression of the A chain/C chain of the platelet-derived growth factor (PDGF), promotes the production of thromboxane and cyclooxygenase-2, and induces the activation of specific mitogen-activated protein kinase (MAPK) pathway, thus the mRNA expression of angiotensinogen increases (20). (II) UA can also enter the endothelial cells of the human umbilical vein through the organic anion transporters and rapidly induce oxidative stress, which in turn activates the local RAAS system and increases the production of Ang II (21).

Another finding in the present study was that in the pAF group, the serum TG and LDL-C levels were significantly higher than those in the SR group, but were significantly lower in the PAF group. These were not difficult to understand. It is currently believed that the risk factors for $\mathrm{AF}$ are basically the same as those for coronary heart disease (22). In the SR stage, TG and LDL-C promote the occurrence of AF through the mechanism of promoting atherosclerosis, which manifests as the increase of TG and LDL-C in the pAF stage. At this stage, the patient noticed the onset of $\mathrm{AF}$ and took the lipid-lowing drugs, but in the PAF stage, it shows a significant decrease in TG and LDL-C.

\section{Acknowledgments}

We would like to acknowledge the hard and dedicated work of all the staff that implemented the intervention and evaluation components of the study.

Funding: This study was funded by Beijing Hepingli Hospital (2019-1-01). The funding body had no role in the design of the study and collection, analysis, and interpretation of data and in writing the manuscript.

\section{Footnote}

Reporting Checklist: The authors have completed the MDAR checklist and the STROBE reporting checklist. Available at: http://dx.doi.org/10.21037/cdt-20-830

Data Sharing Statement: Available at: http://dx.doi. org/10.21037/cdt-20-830

Conflicts of Interest: All authors have completed the ICMJE uniform disclosure form (available at: http://dx.doi. org/10.21037/cdt-20-830). The authors have no conflicts of interest to declare.

Etbical Statement: The authors are accountable for all aspects of the work in ensuring that questions related to the accuracy or integrity of any part of the work are 
appropriately investigated and resolved. The study was conducted in accordance with the Declaration of Helsinki (as revised in 2013). The study was approved by Ethics Committee of Beijing Hepingli Hospital (No. 20190109) and informed consent was taken from all the patients.

Open Access Statement: This is an Open Access article distributed in accordance with the Creative Commons Attribution-NonCommercial-NoDerivs 4.0 International License (CC BY-NC-ND 4.0), which permits the noncommercial replication and distribution of the article with the strict proviso that no changes or edits are made and the original work is properly cited (including links to both the formal publication through the relevant DOI and the license). See: https://creativecommons.org/licenses/by-nc-nd/4.0/.

\section{References}

1. Westerman S, Wenger N. Gender Differences in Atrial Fibrillation: A Review of Epidemiology, Management, and Outcomes. Curr Cardiol Rev 2019;15:136-44.

2. Madan N, Itchhaporia D, Albert CM, et al. Atrial Fibrillation and Heart Failure in Women. Heart Fail Clin 2019;15:55-64.

3. Li LH, Sheng CS, Hu BC, et al. The prevalence, incidence, management and risks of atrial fibrillation in an elderly Chinese population: a prospective study. BMC Cardiovasc Disord 2015;15:31.

4. Gumprecht J, Domek M, Lip GYH, et al. Invited review: hypertension and atrial fibrillation: epidemiology, pathophysiology, and implications for management. J Hum Hypertens 2019;33:824-36.

5. Kawasoe S, Kubozono T, Yoshifuku S, et al. Uric Acid Level and New-Onset Atrial Fibrillation in the Japanese General Population - Longitudinal Study. Circ J 2018;83:156-63.

6. Saxena T, Ali AO, Saxena M. Pathophysiology of essential hypertension: an update. Expert Rev Cardiovasc Ther 2018;16:879-87.

7. Kanellis J, Watanabe S, Li JH, et al. Uric acid stimulates monocyte chemoattractant protein-1 production in vascular smooth muscle cells via mitogen-activated protein kinase and cyclooxygenase-2. Hypertension 2003;41:1287-93.

8. Kirchhof P, Benussi S, Kotecha D, et al. 2016 ESC Guidelines for the management of atrial fibrillation developed in collaboration with EACTS. Eur J Cardiothorac Surg 2016;50:e1-e88.

9. Maharani N, Kuwabara M, Hisatome I. Hyperuricemia and Atrial Fibrillation. Int Heart J 2016;57:395-9.

10. Ndrepepa G. Uric acid and cardiovascular disease. Clin Chim Acta 2018;484:150-63.

11. Pak S, Yatsynovich Y, Valencia D, et al. Serum Uric Acid and Atrial Fibrillation: Meta-analysis. Crit Pathw Cardiol 2018;17:161-6.

12. McMullan CJ, Borgi L, Fisher N, et al. Effect of Uric Acid Lowering on Renin-Angiotensin-System Activation and Ambulatory BP: A Randomized Controlled Trial. Clin J Am Soc Nephrol 2017;12:807-16.

13. Kong P, Christia P, Frangogiannis NG. The pathogenesis of cardiac fibrosis. Cell Mol Life Sci 2014;71:549-74.

14. Liang WY, Liu WW, Liu ML, et al. Serum uric acid level and left ventricular hypertrophy in elderly male patients with nonvalvular atrial fibrillation. Nutr Metab Cardiovasc Dis 2016;26:575-80.

15. Jalife J, Kaur K. Atrial remodeling, fibrosis, and atrial fibrillation. Trends Cardiovasc Med 2015;25:475-84.

16. Bishu K, Deswal A, Chen HH, et al. Biomarkers in acutely decompensated heart failure with preserved or reduced ejection fraction. Am Heart J 2012;164:763-770.e3.

17. Ames MK, Atkins CE, Pitt B. The renin-angiotensinaldosterone system and its suppression, J Vet Intern Med 2019;33:363-82.

18. Brown NJ. Contribution of aldosterone to cardiovascular and renal inflammation and fibrosis. Nat Rev Nephrol 2013;9:459-69.

19. Verdecchia P, Angeli F, Reboldi G. Hypertension and Atrial Fibrillation: Doubts and Certainties From Basic and Clinical Studies. Circ Res 2018;122:352-68.

20. Corry DB, Eslami P, Yamamoto K, et al. Uric acid stimulates vascular smooth muscle cell proliferation and oxidative stress via the vascular renin-angiotensin system. J Hypertens 2008;26:269-75.

21. Mazzali M, Hughes J, Kim YG, et al. Elevated uric acid increases blood pressure in the rat by a novel crystalindependent mechanism. Hypertension 2001;38:1101-6.

22. Cybulska B, Kłosiewicz-Latoszek L. Landmark studies in coronary heart disease epidemiology. The Framingham Heart Study after 70 years and the Seven Countries Study after 60 years. Kardiol Pol 2019;77:173-80.

Cite this article as: Wang XD, Liu J, Zhang YC, Wang Y, Wang Y, Ma D. Correlation between the elevated uric acid levels and circulating renin-angiotensin-aldosterone system activation in patients with atrial fibrillation. Cardiovasc Diagn Ther 2021;11(1):50-55. doi: 10.21037/cdt-20-830 